\title{
App para la distribución y entrega de tortilla en Ixhuatlan del Sureste, Veracruz
}

\section{App or the distribution and delivery of tortillas in Ixhuatlán del Surest, Veracruz}

MORALES-REYES, Eunice $\dagger^{*}$, KATT-MORALES, Alondra, PACHECO-REYES, Jimmy y VAZQUÉZ-HERNÁNDEZ, Rogelio

Universidad Tecnológica del Sureste de Veracruz, Av. Universidad Tecnológica Lote grande, Número 1, sin colonia, Nanchital, Vearcruz

ID $1^{\text {er }}$ Autor: Eunice, Morales-Reyes / ORC ID: 0000-0003-0658-6957, arXiv Author ID: 2836978, Researcher ID Thomson: S-4739-2018, CVU CONACYT ID: 345179

ID $1^{\text {er }}$ Coautor: Luz Alondra, Katt-Morales / ORC ID: 0000-0002-9982-8718, arXiv Author ID: 2417152, Researcher ID Thomson: S-6606-2018, CVU CONACYT ID: 412698

ID $2^{\text {do }}$ Coautor: Jimmy Noe, Pacheco-Reyes / ORC ID: 0000-0003-3225-0664, arXiv Author ID: 534791, Researcher ID Thomson: X-8774-2019, CVU CONACYT ID: 1015431

ID $3{ }^{\text {er }}$ Coautor: Rogelio, Vazquéz-Hernández / ORC ID: 0000-0001-6109-300X, arXiv Author ID: 467405, Researcher ID Thomson: S-6606-2018, CVU CONACYT ID: 947233

DOI: $10.35429 /$ JOCT.2019.10.3.22.27

Recibido 16 de Marzo, 2019; Aceptado 30 Junio, 2019

\section{Resumen}

La tortilla es el alimento con más presencia en los hogares mexicanos, de acuerdo a informes de la Secretaría de Economía y y el Consejo Regulador de la Masa, en México el consumo por persona es de aproximadamente 90 kilogramos de tortilla al año, de tal forma, que existe un mercado potencial dedicado a producir este alimento. Actualmente muchas tortillerías cuentan con el servicio de venta a domicilio por medio de repartidores, sin embargo, es deficiente, derivado de la falta de coordinación y planeación en rutas y horarios fijos en el servicio de los tortilleros, pudiendose observar una oportunidad para mejorar los servicios de venta y entrega, a través de las Tecnologías de la Información. El objetivo del proyecto es desarrollar una aplicación movil que a partir de una solicitud de tortilla, permita facilitar su distribución y entrega a domicilio en la comunidad de Ixhuatlán del Sureste, Veracruz., así mismo, gestionar las tortillerías a partir de un sitio Web, brindando seguridad y facilidad de uso.

Aplicación móvil, Aplicación web, Tortilla

\begin{abstract}
Tortilla is the food with more presence in Mexican homes, according to reports from the Ministry of Economy and the Regulatory Council of the Mass, in Mexico the consumption per person is approximately 90 kilograms of tortillas per year, so, that there is a potential market dedicated to producing this food. Currently many tortillerías have home delivery service through distributors, however, it is deficient, derived from the lack of coordination and planning on fixed routes and schedules in the service of the tortilleros, being able to observe an opportunity to improve services of sale and delivery, through Information Technology. The objective of the project is to develop a mobile application that, based on a tortilla application, makes it possible to facilitate its distribution and home delivery in the community of Ixhuatlán del Sureste, Veracruz. Likewise, manage tortillerías from a website, providing security and ease of use.
\end{abstract}

Mobile application, Web application, Tortilla

Citación: MORALES-REYES, Eunice, KATT-MORALES, Alondra, PACHECO-REYES, Jimmy y VAZQUÉZHERNÁNDEZ, Rogelio. App para la distribución y entrega de tortilla en Ixhuatlan del Sureste, Veracruz. Revista de Tecnologías Computacionales. 2019. 3-10: 23-28

\footnotetext{
* Correspondencia del Autor (Correo electrónico: eunice.morales@utsv.edu.mx)

$\uparrow$ Investigador contribuyendo como primer autor.
} 


\section{Introducción}

De acuerdo a la Asociación de consumidores orgánicos, "México es el principal consumidor de tortilla en el mundo, se estima que es consumida por el $94 \%$ de la población, por lo que el volumen de producción y consumo es cercano a los 12 millones de toneladas de tortillas por año, lo que representa un porcentaje importante entre los productos alimentarios comercializados en el país". (Asociación de Consumidores Organicos, 2018)

Lo anterior permite observar que existen muchos negocios dedicados a proporcionar este alimento, sin embargo, la forma en que lo proveen, no es tan adecuado, ya que su modelo de venta, ha sido el mismo desde hace muchisimos años.

Actualmente las tecnologías de la información, han permitido agilizar los procesos que se realizan en cualquier empresa, en este articulo, se presenta el prototipo de una aplicación movil (App) que permite agilizar la distribución y entrega a domicilio, permitiendo la disminución de tiempo y la satisfacción de los consumidores.

\section{Planteamiento del Problema}

El consumo de la tortilla es primordial en las familias mexicanas, puesto que es consumida en todas las comidas. En la actualidad muchas tortillerías cuentan con el servicio de venta a domicilio por medio de repartidores que se encargan de llevarlo lo más cerca de las casas de los consumidores. Tenemos en cuenta que existe una problemática en la comunidad de Ixhuatlán del sureste el cual podemos observar la falta de coordinación y planeación en rutas y horarios fijos en el servicio de los tortilleros el cual provoca que los consumidores tengan que esperar por un largo tiempo o no lo puedan adquirir.

Por otra parte, al tener esta mala organización en las diferentes tortillerías provoca que los tortilleros lleguen a tener poca ganancia ya que el estar pasando por muchas calles durante todo el día y muchas de las veces no surtir la tortilla, provocan un gran gasto en el consumo de su combustible provocando generar más egresos que ingresos.
En otras palabras, en la actualidad no contamos con un sistema eficaz tanto para los clientes que adquieren el servicio y ni para los distribuidores que realizan las entregas. Es preocupante que muchas personas por la deficiencia de este servicio tengan que dirigirse a las tortillerías para adquirir este producto, por lo tanto, provoca que muchas personas pierdan tiempo. Esta problemática de la comunidad de Ixhuatlán del sureste ha prevalecido por mucho tiempo y no se le ha dado la importancia que este requiere.

También es signifivativo mencionar el problema del retraso que algunas tortillerías presentan a la hora de la elaboración y entrega de tortilla, ya que muchas veces este proceso suele ser muy lento, lo cual provoca retraso y disgusto para las personas que se encargan de entregar este servicio y para las que están esperando su turno para adquirir tortilla. En conclusión, es indispensable utilizar las tecnologías de la información para mejorar los servicios de distribución y entrega de tortilla en el municipio de Nanchital, Veracruz.

\section{Justificación}

Por la deficiencia del servicio en las entregas de tortillas en la comunidad de Ixhuatlán del sureste en el Estado de Veracruz, se desarrolla una aplicación movil, encargada de llevar un orden en la entrega de tortilla. Mediante está se pretendé que la relación entre cliente y servicio sea más amigable, confiable y segura.

Esta App se encargara de mejorar el servicio actualmente ofrecido por los repartidores, de tal forma que la entrega a domicilio sea más rápida. El cliente al tener la aplicación móvil podrá enviar una notificación solicitando el servicio, el cual será el requerimiento de tortilla, que a su vez se les notificará a los tortilleros que estén dados de alta en esta plataforma con la ubicación actual del usuario. Esto permitirá que el tortillero más cercano solicite llevar esa entrega avisando al usuario que su tortilla está en camino. De este modo las personas contaran con un servicio confiable el cual no les provocara asistir a una tortillería, haciéndoles perder tiempo.

\section{Objetivo General}

Desarrollar una aplicación móvil que facilite la distribución y entrega de tortillas a domicilio en la comunidad de Ixhuatlán del sureste.

MORALES-REYES, Eunice, KATT-MORALES, Alondra, PACHECOREYES, Jimmy y VAZQUÉZ-HERNÁNDEZ, Rogelio. App para la distribución y entrega de tortilla en Ixhuatlan del Sureste, Veracruz. Revista de Tecnologías Computacionales. 2019 


\section{Metodología}

Para lograr que el desarrollo de la aplicación sea de acuerdo a estandarés de calidad, se incorporó la metodologia ágil Scrum, desarrollada por Ken Schwaber y Jeff Sutherland, quienes detallan en la guía definitiva de Scrum ${ }^{\mathrm{TM}}$, que está "es un marco de trabajo de procesos que ha sido usado para gestionar el desarrollo de productos complejos desde principios de los años 90. Scrum no es un proceso o una técnica para construir productos; en lugar de eso, es un marco de trabajo dentro del cual se pueden emplear varios procesos y técnicas. Scrum muestra la eficacia relativa de las prácticas de gestión de producto y las prácticas de desarrollo de modo que podamos mejorar". (Sutherland, 2016)

El marco de trabajo Scrum consiste en los Equipos Scrum y sus roles, eventos, artefactos y reglas asociadas. Cada componente dentro del marco de trabajo sirve a un propósito específico y es esencial para el éxito de Scrum y para su uso. En la figura 1 se puede observar el marco de trabajo de la metodología.

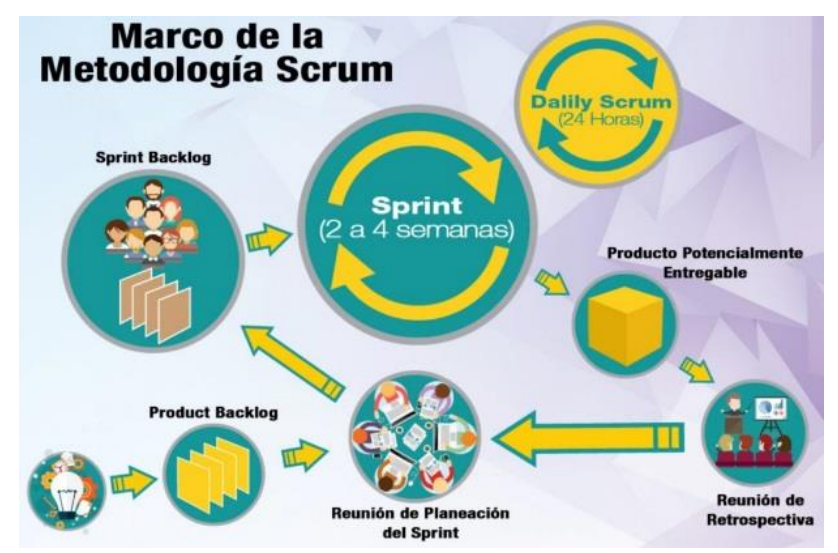

Figura 1 Marco de la metodología Scrum ${ }^{\mathrm{TM}}$

\section{Desarrollo}

\section{Factibilidad del Proyecto}

Con el objetivo de realizar los estudios de factibidad de proyecto, se diseño una encuesta utilizando una metodología exploratoria para obtener los tiempos, costos y alcance del proyecto estudiado, con esta metodología se consigue una medición que conlleva a información fidedigna y válida para de esta manera tomar decisiones con base en los resultados generados por el estudio.
Se aplicarón 25 encuestas a expendios de tortilla para medir el grado de interés en implementar las tecnologías de la información y optimizar con esto su modelo de servicio al cliente, en relación a la entrega de tortilla a domicilio.

Los resultados fuerón los siguientes: El $55 \%$ de las tortillerías del municipio ofrecen servicio a domicilio, de las cuales en promedio tienen 2 tortilleros repartidores, el $95 \%$ de las tortillerías encuestadas están interesadas en el servicio de TortiX para la repartición a domicilio de las tortillas, así mismo, en la capacitación de sus empleados para el uso de la aplicación.

\section{- $\quad$ Requisitos Funcionales \\ - Sitio Web}

- Gestionar tortillerías: Este apartado web, permitirá al administrador, agregar, modificar, consultar y eliminar las tortillerías que tienen activo el servicio de entrega.

- Gestionar tortilleros: Este apartado web, permitira a las tortillerías, agregar, modificar, consultar y eliminar a los tortilleros que estan a su cargo, y que realizaran la entrega a partir de una solicitud.

- Gestionar usuarios: Este apartado web, permitira al administrador dar de alta, eliminar, consultar y modificar a los usuarios que utilizaran el servicio movil para la solicitud de tortilla a domicilio.

Consultar información de tortillerías: Este requerimiento permitirá a los usuarios conocer informacion como los horarios, rutas, etc., de las tortillerías.

\section{- $\quad$ Aplicación Movil}

- $\quad$ Iniciar Sesión: Los usuarios y tortilleros deben iniciar sesión para poder usar el servicio.

- Solicitud de tortilla: Este apartado permitirá a los usuarios solicitar un pedido de tortilla indicando el numero de Kg.

- Notificación al tortillero más cercano donde se especificará la ubicación del usuario, para gestionar la entrega. 


\section{Diagrama de Casos de Uso}

A continuación, se muestra el diagrama de casos de usos del sitio web que administrará el sistema TortiX utilizando UML (Ver figura 2).

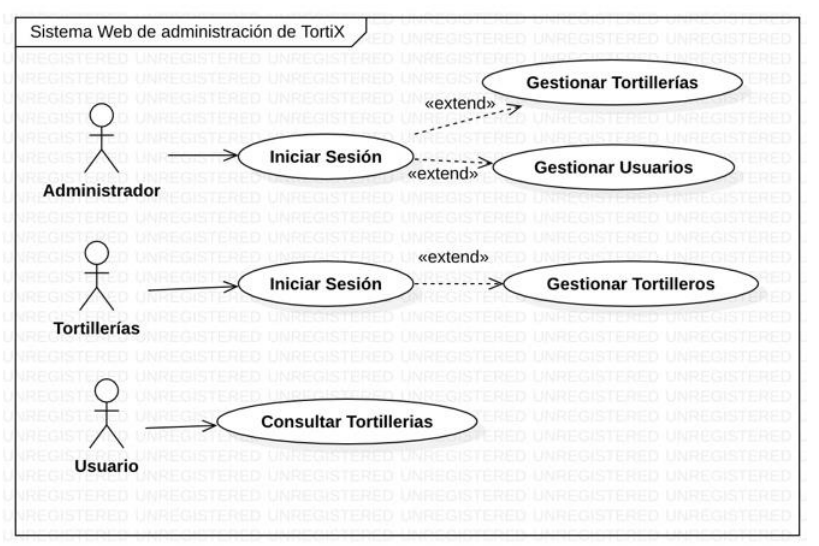

Figura 2 Diagrama de Casos de Uso Sitio Web

En la figura 3, se puede observar el diagrama de casos de uso, de la aplicación movil, donde se muestran las acciones que podrán realizar los usuarios en el sistema.

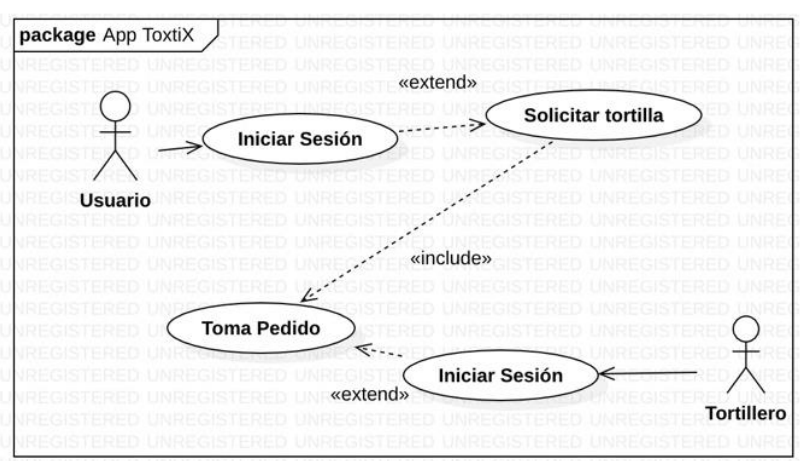

Figura 3 Diagrama de Casos de Uso del Sitio Movil

\section{Resultados}

\section{- Interfaz gráfica del Sistema Web de administración de ToxtiX}

En la figura 4 se observa la interfaz principal del sistema Web de TortiX, este sitio online permitirá administrar los datos de las tortillerías que estén interesadas en el servicio, estás podrán contar con un usuario y contraseña (Ver figura 5) para poder gestionar la información que de publicidad a su negocio, tales como, productos que ofrece, horarios de servicio, etc., asi mismo, podrá agregar a los tortilleros que harán llegar las tortillas al domicilio del cliente, cabe mencionar que para su seguridad, estos deben contar con requisitos para poder ofrecer el servicio, por los datos que se manejan.

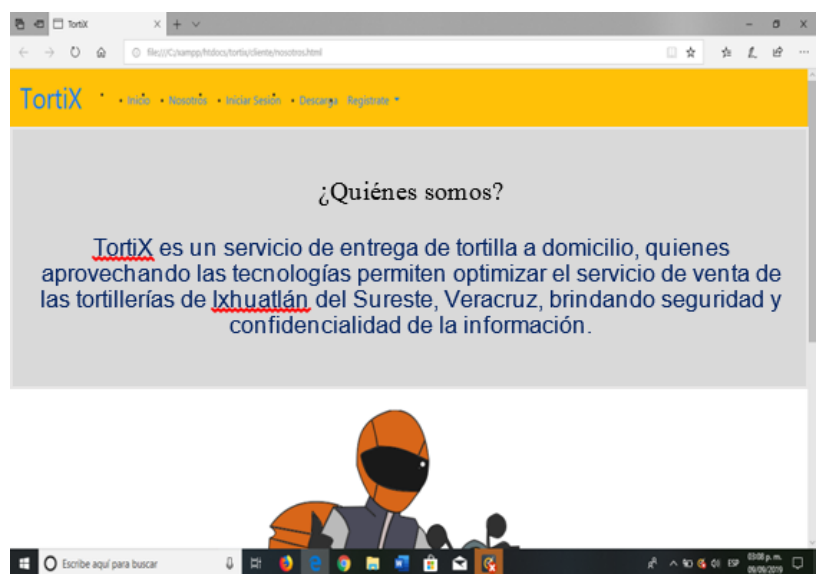

Figura 4 Interfaz gráfica Nosotros- Página Principal

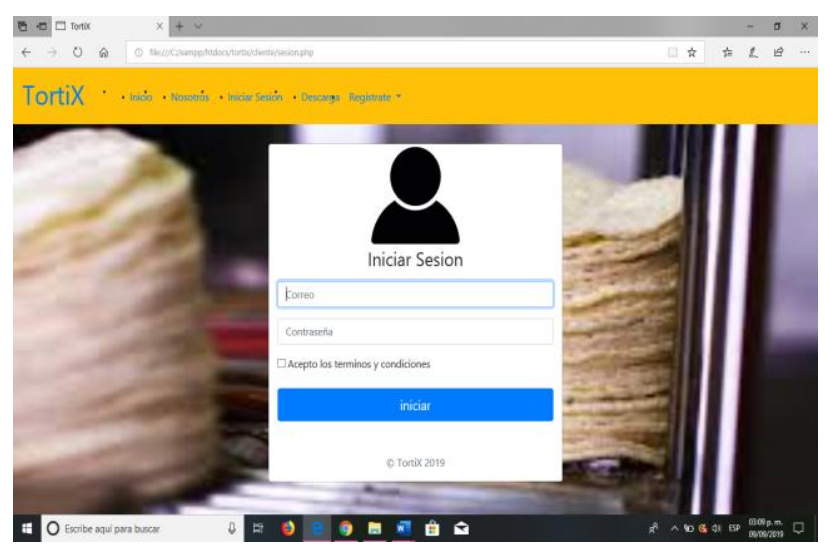

Figura 5 Interfaz gráfica Iniciar sesión

En la Figura No. 6 se puede observar la interfaz gráfica de la página que permitira el registro de los usuarios.

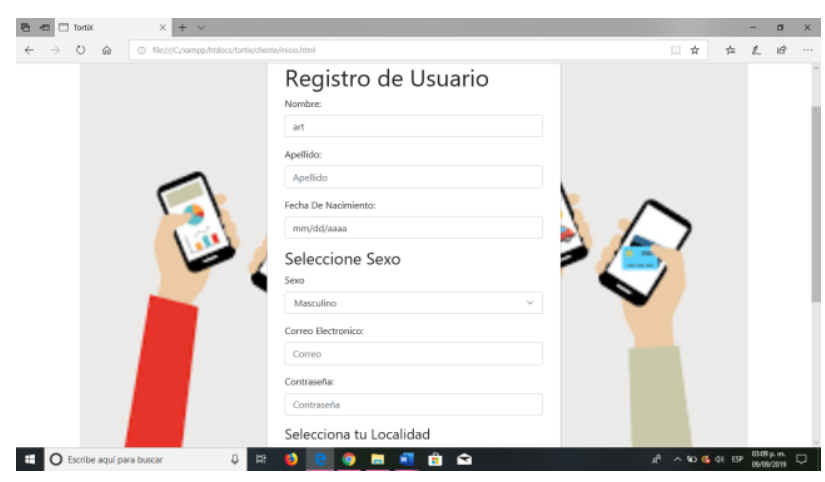

Figura 6 Interfaz gráfica Registro de Usuario

\section{Interfaces gráficas de la App}

La aplicación movil de TortiX permitirá a los clientes solicitar pedidos de tortillas, siempre y cuando esten registrados en el sitio web (Ver Figura 6), estos deberán loguearse, tal como aparece en la figura 7 . 


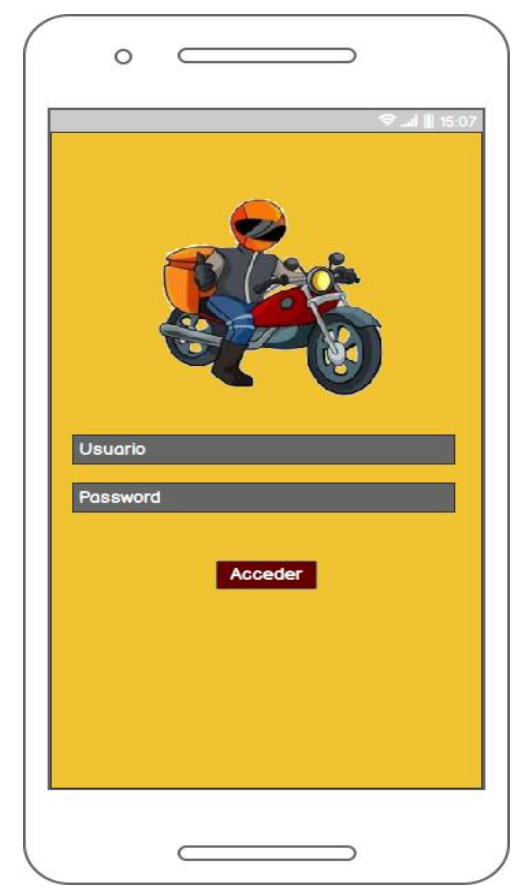

Figura 7 Interfaz móvil - Iniciar sesión

En la Figura 8 se puede observar la interfaz que permitirá al usuario solicitar los pedidos de tortilla a los tortilleros disponibles a quienes les llegará la solicitud de servicio indicando la ubicación y los datos del pedido. (Ver Figura 9).

Es importante mencionar que estás recepciones de pedido, se enviaran a los tortilleros que se encuentren más cerca, lo que permitira optimizar el tiempo de la entrega.

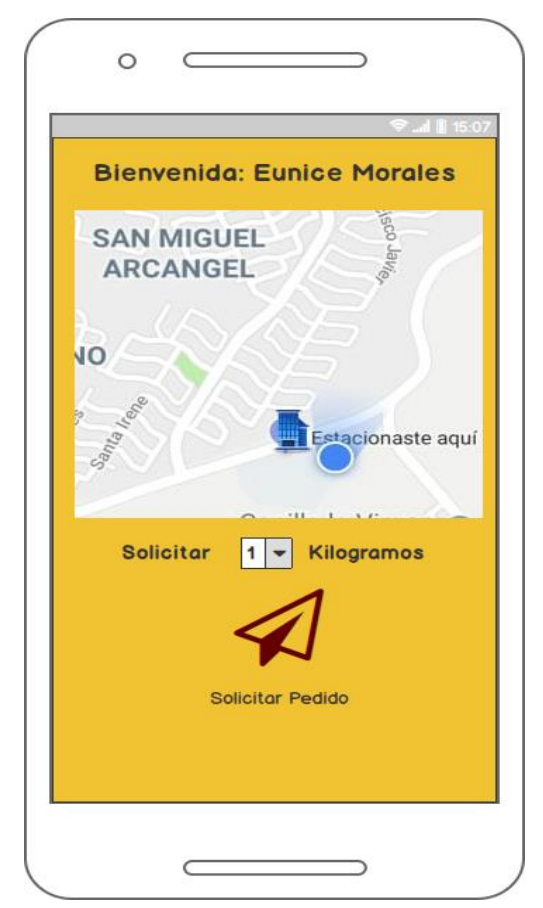

Figura 8 Interfaz móvil - Solicitud de Pedido

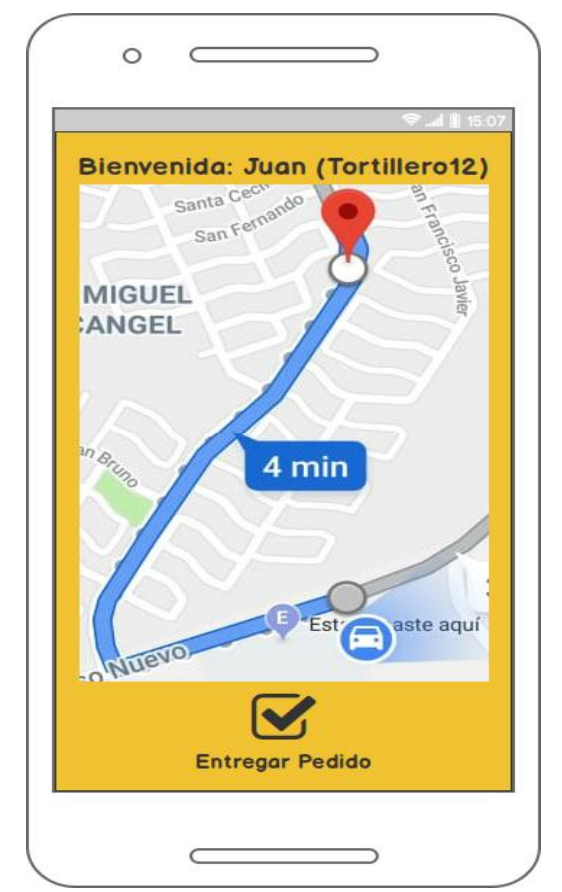

Figura 9 Interfaz móvil - Recepción de Pedido

Una vez que se le notifica al tortillero más cercano, este responde la solicitud de entrega, enviando al usuario una notificacion de que el pedido se encuentra en camino. (Ver figura 10)

El usuario podrá dar seguimiento a la ruta del tortillero en tiempo real vía Google Maps, facilitando con esto la forma en la que se entrega la tortilla. Actualmente la aplicación movil, solo se desarrollará para la tecnología Android, puesto que es la tecnología movil actualmente más utilizada, dejando posteriormente el desarrollo en IOS.

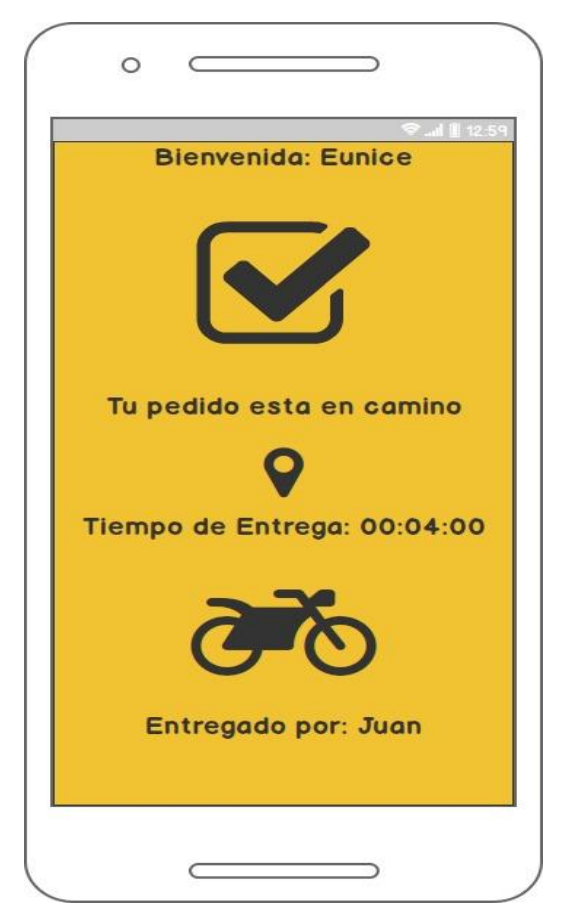

Figura 10 Interfaz móvil - Notificación de entrega

MORALES-REYES, Eunice, KATT-MORALES, Alondra, PACHECOREYES, Jimmy y VAZQUÉZ-HERNÁNDEZ, Rogelio. App para la distribución y entrega de tortilla en Ixhuatlan del Sureste, Veracruz. Revista de Tecnologías Computacionales. 2019 


\section{Conclusiones}

La tecnología juega un papel sumamente importante en casi todos los ámbitos de nuestras vidas, ha sido capaz de transformar el estilo de vida de los seres humanos de tal manera que sería difícil imaginar un día sin ella.

Con el desarrollo e implementacion de la aplicación TortiX, los negocios de tortilla permitirán mejorar la accesibilidad de sus productos, logrando con esto, la optimizacion de sus entregas a domicilio, y el control de sus usuarios, de tal forma, que su modelo de negocio se vuelva más eficiente, incrementando con esto, sus ganancias.

Asi mismo se espera que los usuarios que usen este servicio, esten satisfechos con la forma en que realizan la compra a los expendios de tortilla, logrando con esto, la innovacion en los servicios, optimizando el tiempo de compra.

Es importante mencionar que el desarrollo de este tipo de aplicaciones, permitira a las tortillerías de Ixhuatlan del Sureste, eplantear e integrar sus procesos, reducir costos, mejorar las comunicaciones, transformar los procesos de negocio e implementar una cultura sólida.

\section{Referencias}

Asociación de Consumidores Organicos. (2018). Obtenido de https://consumidoresorganicos.org: https://consumidoresorganicos.org/2018/01/15/ sube-la-tortilla-pero-no-su-calidad/

Balado, E. S. (2005). Estrategia para la implantación de nuevas tecnologías en PYMEs. Obtenga el máximo rendimiento aplicando las TIC en el ámbito empresarial. Ideaspropias Editorial.

González, J. A. (2016). Reingeniería de procesos empresariales. Fundación Confemetal.

OCDE. (2013). Temas y políticas clave sobre PYMEs y emprendimiento en México. OECD Publishing.

Sutherland, K. S. (2016). La Guía de ScrumTM. Obtenido de www.scrum.org: https://www.scrumguides.org/docs/scrumguide/ v2016/2016-Scrum-Guide-

Spanish.pdf\#zoom=100 